\title{
Factores condicionantes en el desarrollo de episodios depresivos en universitarios: Una perspectiva desde Dorothea Orem
}

\author{
Teresita de Jesús Muñoz Torres, * Rocío Rocha Rodríguez,** Milka Elena Escalera Chávez,*** \\ María Yolanda Méndez Bernal*
}

\begin{abstract}
RESUMEN
Objetivo: Determinar los factores condicionantes en el desarrollo de episodios depresivos en universitarios, a partir de los elementos básicos considerados en la Teoría General del Déficit de Autocuidado de Dorothea Orem. Metodología: Estudio cuantitativo, prospectivo, descriptivo, correlacional-causal y de corte transversal. Muestra de 819 alumnos de los diversos semestres y carreras (Licenciatura en Enfermería, Licenciatura en Administración, Contador Público, Ingeniero Civil y Licenciatura en Mercadotecnia). Se utilizó un instrumento creado exprofeso para medir los diversos factores, con un alfa de Crombach de 0.92. El procesamiento estadístico se realizó a través del programa SPSS versión 17, utilizándose análisis descriptivo y regresión lineal logística. Resultados: El 14.6\% de las universitarias presentan episodios depresivos, mientras que en los estudiantes de género masculino este padecimiento se presenta en un 5.7\%. Los factores condicionantes básicos identificados por medio del análisis cuantitativo son los sociodemográficos $(\mathrm{p}=0.002)$, estado de salud $(\mathrm{p}=0.040$, promedio) y ambiente ( $\mathrm{p}=0.000)$. Conclusión: Se brinda un panorama de acción diferente y complementario, para proporcionar una atención de calidad a partir de la prioridad de Orem, que es controlar y dirigir los factores hacia un equilibrio biopsicosocial y espiritual.
\end{abstract}

Palabras clave: Depresión, universitarios, factores condicionantes, Dorothea Orem.

\section{Conditioning factors in the development of depressive episodes in University: A perspective from Dorothea Orem}

\begin{abstract}
Objective: Determine the factors in the development of depressive episodes in academics, from the basic elements considered in the General Self-Care Deficit Theory of Dorothea Orem. Methodology: Quantitative, prospective, descriptive, correlacional-causal and transversal study. Sample of 819 students of the various semesters and courses (Nursing, Administration, Public Accountant, Civil Engineer and Marketing). I will use an instrument created by the authors for measuring the various factors, with an Crombach alpha of 0.92. Statistical processing carried out through the program SPSS version 17, using descriptive analysis and logistic linear regression. Results: The 14.6\% of the University have depressive episodes, while in the male students this condition presents in a 5.7\%. The conditioning basic factors identified by the quantitative analysis were the sociodemographic $(\mathrm{p}=0.002)$, health $(\mathrm{p}=0.040$, average $)$ and environment $(\mathrm{p}=0000)$. Conclusion:
\end{abstract}

\footnotetext{
* Estudiante de la Licenciatura en Enfermería.

** Doctora en Ciencias de Enfermería. Profesora investigadora de tiempo completo de la Licenciatura en Enfermería.

*** Doctora en Administración. Profesora Investigadora de tiempo completo de la Licenciatura en Administración.
}

Unidad Académica Multidisciplinaria Zona Media de la Universidad Autónoma de San Luis Potosí, México.

Correspondencia: Teresita de Jesús Muñoz Torres. Cerrada de Pirules Núm. 207. UHI. Ojo de Agua, Rioverde, San Luis Potosí. 4871121299. E mail: teresita._torres@hotmail.com

Este artículo puede ser consultado en versión completa en http://www.medigraphic.com/enfermerianeurologica 
Provides an overview of action different and complementary, to provide quality care as the priority of Orem, which is to control and direct factors towards an equilibrium biopsychosocial and spiritual.

Key words: Depression, University, conditioning factors, Dorothea Orem.

\section{INTRODUCCIÓN}

$\mathrm{D}$ orothea Orem enmarcaba en su teoría del déficit del autocuidado que la capacidad de autocuidado terapéutico es el conjunto de medidas adecuadas para cubrir las necesidades de una persona; a su vez definía que para formular una atención de calidad centrada en el modelo es necesario utilizar métodos óptimos con la finalidad de controlar y dirigir los factores identificados en las necesidades para mantener un equilibrio dentro de una gama de valores; ${ }^{1}$ dichos factores son denominados condicionantes básicos, entre los que se encuentran los demográficos, estado de desarrollo, ambiente, familia, factor social y cultural, religión, recurso financiero y estado de salud, cada uno con sus respectivos indicadores, los que para este estudio serán identificados como variables.

La depresión es definida como el conjunto de síntomas que interfieren con la capacidad de los individuos para disfrutar de actividades que, en situaciones normales, deberían ser placenteras; se presenta en más de 150 millones de personas a nivel mundial, según la Organización Mundial de la Salud (OMS); ${ }^{2}$ de esta cifra, 20\% de los jóvenes la padecen convirtiéndose en la $1^{\text {a }}$ causa de suicido entre este grupo de edad; ${ }^{3}$ por su parte, Santillán estima que la depresión es la $2^{\mathrm{a}}$ causa de discapacidad a nivel mundial con población total de 450 millones; ${ }^{4}$ En México, la Secretaría de Salud refiere que de un 12 a $20 \%$ de la población de 18 a 65 años padece depresión o la sufrirá en algún momento de su vida; ${ }^{5}$ sin embargo, el Instituto Nacional de Estadística, Geografía e Informática, afirma que en México hay 20,660,000 mexicanos que padecen depresión, es decir, el $17 \%$ de la carga mundial. ${ }^{6}$ Por su parte, Loredo Maldonado, investigadora de la Universidad Autónoma de San Luis Potosí, reportó que un $40.6 \%$ de los estudiantes de esta institución padecen de episodios depresivos y que este problema de salud mental se presenta en un rango de 17 a 24 años (depresión leve), mientras que la depresión severa se presenta de los 25 a los 29 años.

Varios autores y dependencias de salud enmarcan algunos factores clave en el desarrollo de episodios depresivos; por ejemplo, la OMS estipula que un gran porcentaje de las personas con enfermedades físicas-crónicas tienden a desarrollar estados depresivos; ${ }^{2}$ Bermeo Méndez establece que las enfermedades crónico-degenerativas (respiratorias, diabetes, cardiovasculares) tienen una estrecha relación con las enfermedades mentales, porque el proceso patológico las vuelve más vulnerables. ${ }^{4}$
La Encuesta Nacional Epidemiológica de Psiquiatría refirió que los trastornos afectivos como la depresión prevalecen más en el sexo femenino (6-11.8\%) en comparación con los varones (5.5\%) debido a los factores genéticos y hormonales, convirtiéndola en la $4^{\mathrm{a}}$ enfermedad médica más costosa. ${ }^{6,8}$

Las teorías conductuales de la depresión están estrechamente relacionadas con la indefensión aprendida; esta teoría establece que la depresión es causa-efecto de la falta de refuerzo de conductas no depresivas, generando un déficit de conductas adaptativas sociales como lo es la asertividad, la solución-respuesta a desafíos y la búsqueda de reforzadores como la emotividad, los cuidados y la atención. ${ }^{8}$

Lo antes planteado refuerza la formulación de la OMS en la que deja ver, a través de las investigaciones recientes, que la depresión puede y debe ser tratada en los marcos de una atención primaria; a su vez, resalta el objetivo de este estudio que es determinar cuál de las variables independientes que se encuentran dentro del contexto de la teoría de Dorothea Orem influyen en la probabilidad del desarrollo de episodios depresivos, así como la presencia significativa o no de los factores condicionantes básicos.

\section{MATERIAL Y MÉTODOS}

Es una investigación cuantitativa, descriptiva, correlacionalcausal y de corte transversal, realizada en la Unidad Académica Multidisciplinaria Zona Media de la Universidad Autónoma de San Luis Potosí. Se aplicó censo poblacional, obteniéndose una muestra total de 819 alumnos inscritos a las carreras de Enfermería, Contador Público, Ingeniero Civil, Mercadotecnia y Administración. El estudio se llevó a cabo de agosto a noviembre de 2010. Se incluyeron a los alumnos inscritos en las diversas carreras, se eliminó a la población que se negó a participar en el estudio y no se aplicaron criterios de exclusión. La prueba piloto se aplicó a una muestra total de 21 alumnos y se utilizó como instrumento de recolección de datos el cuestionario Programa de Promoción a la Salud, creado por los autores y el cual mostró un Alpha de Crombach de 0.92 calculado mediante la varianza de los ítems. Dicho instrumento está constituido en su primera parte por ítems y dirigido a identificar las características sociodemográficas de los alumnos (edad, género, estado civil, ocupación, carrera e hijos); en su segundo apartado, se exploran diversas áreas relacionadas a la salud de los estudiantes universitarios como 
es la alimentación, actividad física, salud sexual, adicciones, problemas alergénicos, sensorio-motrices, enfermedades físico-crónicas, así como la presencia de estados depresivos; cada ítem es evaluado con una escala diferente; sin embargo, para el análisis de este estudio se utilizaron variables de respuesta binomialmente distribuidas.

Para el procesamiento estadístico de los datos se utilizó el Statistical Package for the Social Sciences (SPSS) versión 17.0, aplicándose frecuencias, promedios, porcentajes, desviación estándar y tablas de contingencia para la estadística descriptiva; para el análisis correlacional-causal de las variables independientes y dependientes se utilizó una regresión lineal logística.

\section{RESULTADOS Y ANÁLISIS}

Se realizó un análisis descriptivo para determinar las características de la muestra objeto de estudio, las cuales se encuentran representados en el cuadro I.

Para determinar la presencia o ausencia de los factores condicionantes básicos que intervienen en el desarrollo de episodios depresivos en universitarios según el modelo de Dorothea Orem, se utilizó un análisis correlacional-causal de cada una de las variables representadas en el cuadro I a través de una regresión lineal logística; los resultados obtenidos se encuentran representados en el cuadro II.

Lo anterior permite establecer que los factores condicionantes básicos intervienen en el desarrollo de los episodios depresivos en universitarios; es decir, las variables contempladas en cada uno de los factores condicionantes: los demográficos intervienen en un 3\%, el estado de salud en un $16.4 \%$ y los ambientales en un $6.6 \%$. También establece que la violencia intrafamiliar se asocia con los episodios depresivos 16 veces más en relación con los problemas de salud presentes en la muestra objeto de estudio.

\section{DISCUSIÓN Y CONCLUSIONES}

Los hallazgos de la presente investigación muestran que el sexo, problemas alergénicos, psicomotrices, visuales y violencia intrafamiliar reflejan una significativa influencia

\begin{tabular}{|c|c|c|c|c|}
\hline Característica & Indicador & $\%$ & Factor condicionante básico & Tipo de variable \\
\hline Carrera & $\begin{array}{l}\text { Enfermería } \\
\text { Administración } \\
\text { Ingeniería civil } \\
\text { Mercadotecnia } \\
\text { Contador público }\end{array}$ & $\begin{array}{l}21.4 \\
24.8 \\
18.1 \\
15.3 \\
20.5\end{array}$ & Factor social & \\
\hline $\begin{array}{l}\text { Episodio depresivo } \\
\text { presente por carrera }\end{array}$ & $\begin{array}{l}\text { Enfermería } \\
\text { Administración } \\
\text { Ingeniería civil } \\
\text { Mercadotecnia } \\
\text { Contador público }\end{array}$ & $\begin{array}{c}18.8 \\
8.3 \\
6 \\
11.2 \\
12.5\end{array}$ & y cultural & \\
\hline Sexo & $\begin{array}{l}\text { Masculino } \\
\text { Femenino }\end{array}$ & $\begin{array}{l}35.9 \\
64.1\end{array}$ & Demográficos & \\
\hline Edad & \multicolumn{2}{|l|}{$\begin{array}{l}17 \text { años a } 38 \text { años; promedio } \\
\text { de } 20.3 \text { años y } D E \pm 2.3\end{array}$} & & \\
\hline Estado civil & $\begin{array}{l}\text { Soltero } \\
\text { Casado } \\
\text { Unión libre }\end{array}$ & $\begin{array}{r}95.6 \\
2.0 \\
2.4\end{array}$ & Familia & Independiente \\
\hline Hijos & $\begin{array}{l}\text { Sí } \\
\text { No }\end{array}$ & $\begin{array}{r}4.5 \\
95.5\end{array}$ & & \\
\hline Situación laboral & $\begin{array}{l}\text { No } \\
\text { Sí }\end{array}$ & $\begin{array}{l}80.8 \\
19.2\end{array}$ & & \\
\hline Ingreso económico & $\begin{array}{l}\$ 500-1000 \\
\$ 1001-1500\end{array}$ & $\begin{array}{l}42 \\
16.4\end{array}$ & Recurso financiero & \\
\hline Dependencia económica & $\begin{array}{l}\text { Padres } \\
\text { Otro familiar }\end{array}$ & $\begin{array}{l}85.6 \\
14.4\end{array}$ & & \\
\hline Adicciones & $\begin{array}{l}\text { Alcohol } \\
\text { Tabaco }\end{array}$ & $\begin{array}{l}44.8 \\
10.3\end{array}$ & Ambiente & \\
\hline Violencia intrafamiliar & $\begin{array}{l}\text { Sí } \\
\text { No }\end{array}$ & $\begin{array}{r}1.6 \\
98.4\end{array}$ & & \\
\hline Patologías & $\begin{array}{l}\text { Crónico-degenerativas } \\
\text { Problemas visuales } \\
\text { Psicomotriz, psicológico o psiquiátrico } \\
\text { Alergias }\end{array}$ & $\begin{array}{r}1.3 \\
22.7 \\
1.6 \\
13.9\end{array}$ & Estado de salud & \\
\hline Vida sexual activa & Femenino & $\begin{array}{r}31.4 \\
68.6 \\
\text { lino }\end{array}$ & Estado de desarrollo & \\
\hline $\begin{array}{l}\text { Presencia de episodios } \\
\text { depresivos }\end{array}$ & $\begin{array}{l}14.6 \% \\
85.4 \%\end{array}$ & & Estado de salud & Dependiente \\
\hline
\end{tabular}

Fuente: Elaboración propia 
Cuadro II. Análisis de regresión lineal logística.

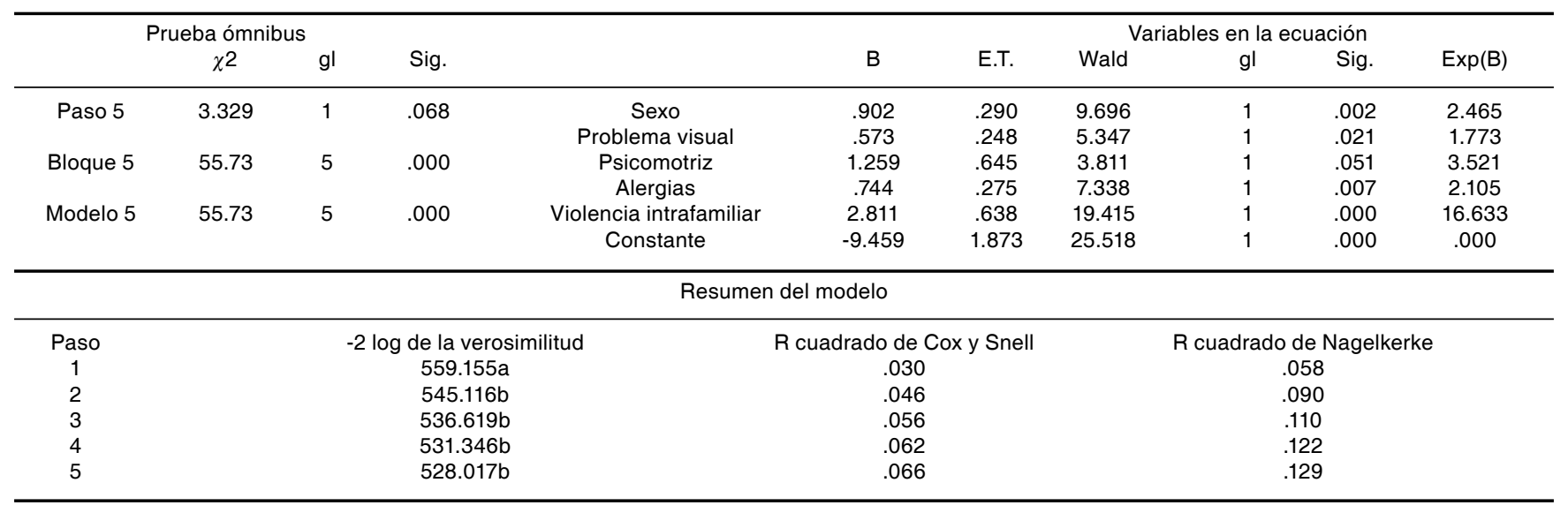

Sig. (Significancia); gl (grados de libertad); $\chi 2$ (Chi-cuadrada): E.T. (error estándar).

Fuente: Elaboración propia

sobre la presencia de episodios depresivos en los jóvenes universitarios de las diversas carreras de la Unidad Académica Multidisciplinaria Zona Media de la Universidad Autónoma de San Luis Potosí.

Los resultados demuestran que el género de los universitarios es determinante en la presencia o no de los episodios depresivos, pudiendo atribuir esto a factores psicológicos, genéticos o sociales. Arrivillaga y cols. enmarcan que si bien lo anterior pudiera ser significativamente importante, también las mujeres son propensas a desarrollar esta patología por situaciones biológicas como los cambios hormonales, estrés, responsabilidades, exceso de trabajo como parte de un rol natural, en comparación con el papel masculino en donde sus procesos biológicos y sociales son menos marcados, ya que al análisis de una mayor vulnerabilidad se encontró que mientras las mujeres suelen dirigirse hacia un manejo de emociones, los hombres se centran en la solución del problema. ${ }^{9-11}$

Se considera que el desarrollo de los episodios depresivos está siempre ligado con la edad de la población; sin embargo, el presente estudio establece que en los alumnos de la Unidad Académica Multidisciplinaria Zona Media esto no se manifiesta así; por su parte, un análisis sobre el diagnóstico de depresión en la población mexicana encontró una estrecha relación con la edad; de la misma manera, en un estudio realizado en Colombia se identificó que los episodios depresivos tienden a aumentar con la edad, pero no fue posible demostrar la presencia de un comportamiento similar al de esta investigación con otros estudios de esta índole..$^{12,13}$ Con relación a las variables económicas, varios autores señalan que un porcentaje de los alumnos con episodios depresivos ha sufrido en los últimos meses inestabilidad económica, con lo que se establece que situaciones estresantes podrían ser generadoras de episodios depresivos; ${ }^{9} \sin$ embargo, para el presente estudio esto no resultó estadísticamente significativo, puesto que la mayoría de los alumnos son económicamente dependientes de los padres, hermanos o tíos, y no sienten una amenaza latente que les implique un desempleo, problemas laborales o severas condiciones de trabajo, situaciones laborales que afectan el estado de ánimo. Dentro del análisis se pretendía determinar la importancia de la carrera profesional en función de los episodios depresivos; no obstante, ninguna de las carreras resultó ser significativa. A pesar de ello, varios autores establecen que la profesión de enfermería tiende a desarrollar más episodios depresivos por estar en contacto con estresores dentro de su ámbito, como son la falta de competencia, la relación docente-alumno-compañeros durante la práctica, la incapacidad para controlar la relación alumna-paciente, involucrarse emotivamente con los pacientes y la sobrecarga de trabajo, ${ }^{14} \sin$ embargo, al revisar la literatura no se encontraron estudios que aceptaran o rechazaran la significancia entre las carreras de nivel profesional (mercadotecnia, contador público, ingeniería civil y administración) y la depresión. Respecto a las variables de estado civil e hijos, éstas no representan un factor de interés para establecerlas como determinantes en la presencia de estados depresivos, datos que Arias en su estudio respalda al establecer que tales características no permiten crear una diferenciación para determinar las posibles causas de episodios depresivos; ${ }^{15}$ sin embargo, algunos autores establecen que la presencia de depresión está íntimamente ligada a estructura y composición de un hogar, así como el estado civil. ${ }^{13}$

En las últimas décadas, la presencia de episodios depresivos ha estado estrechamente relacionada con el consumo de sustancias como alcohol y tabaco, en donde a la revisión de las historias clínicas de los pacientes, las personas deprimidas tienden a consumirlas más, ${ }^{13,16} \sin$ embargo, esto no aplica 
para los universitarios en estudio, dado que el consumo de estas sustancias está más vinculado con conductas de nivel social (amistad, convivencia de pares).

Los estados de salud como los problemas visuales, enfermedades alergénicas y psicomotrices fueron importantes en el desarrollo de episodios depresivos en universitarios, debido a que afectan su desempeño y actividades pues condicionan en cierto grado su participación dentro de un contexto social por temor al rechazo o disminución de su autoestima; sin embargo, en la literatura no se encuentran estudios que aborden dichos aspectos, por lo que no se puede confirmar o rechazar dicho análisis.

Si bien se ha hablado de diversos factores, el más importante es la violencia dentro del hogar como desencadenante de los episodios depresivos, pues ésta no sólo afecta el desenvolvimiento individual, la capacidad de concentración y la respuesta a los estresores, sino que origina que la persona pierda el ambiente social, mismo que le permite mantener su equilibrio biológico, psicológico y social; varias investigaciones han encontrado que la buena relación entre padres e hijos y la convivencia sana dentro del hogar nuclear forman un factor protector en relación con la depresión. ${ }^{17}$ Castillo Manzano, en su investigación determina una relación significativa entre el ser mujer, violencia intrafamiliar y episodios depresivos, al identificar en sus grupos control y no control estos aspectos ${ }^{18}$ lo que demuestra lo identificado en este estudio, al establecer que los episodios depresivos son más frecuentes en la mujer y en quien padece violencia dentro del núcleo familiar.

A modo de conclusión, y teniendo en cuenta su metodología, este estudio permite crear una asociación entre lo que se sabe y lo que se hace, pues facilita vincular la acción de enfermería con los programas de atención en materia de salud; también le presenta al profesional de enfermería y a los alumnos en formación profesional un panorama de acción diferente pero a la vez complementario, considerando que para brindar una atención de calidad y libre de riesgos se necesita partir de la prioridad que sugiere el modelo de Orem, que es controlar y dirigir los factores que determinan las necesidades del ser humano y que son el pilar del equilibrio biopsicosocial y espiritual, permitiéndole un alto grado de eficiencia vinculada con la tecnología y las técnicas, teniendo en cuenta que para el individuo son indispensables los estímulos del entorno para sobrevivir de acuerdo al dinamismo de la naturaleza misma.

\section{BIBLIOGRAFÍA}

1. Marriner TA, Raile AM. Modelos y teorías en enfermería. $6^{a}$ edición. España: Editorial Elsevier, 2007.

2. Organización Mundial de la Salud. Informe sobre la salud en el mundo. Capítulo 1: Salud mundial: retos actuales. 2003; [10 páginas aprox.]. Disponible en: URL http://www.who.int/whr/2003/chapterl/es/index3. html. Consultado marzo 8, 2011.

3. Elizalde M. La depresión es la primera causa de suicidio en jóvenes. Debate.com.mx 2010 Octubre 10; Sec. General.

4. Santillán L. Depresión, segunda causa de discapacidad a nivel mundial. Diario Portal 2010 Octubre 6; Sec. Portada.

5. Lira SC. La depresión, enfermedad del siglo XXI, afecta en México a 10 millones. La Jornada 2005 Febrero 23; Sec. Ciencias.

6. Alberga México 17\% de personas con depresión en el mundo. El universal. com.mx 2008 Mayo 15; Sec. Salud.

7. Briones ZP. El $40.6 \%$ de universitarios sufre de estados depresivos. El Sol de San Luis 2008, enero 20; Sec. Salud.

8. Hales R, Yudofsky S. Tratado de psiquiatría clínica. $4^{a}$ edición. España: Editorial Masson, 2004.

9. Arrivillaga QM, Cortés GC, Goicochea JV, Lozano OT. Características de la depresión en jóvenes universitarios. Univ Psychol Bogotá (Colombia) 2004 ; 3 (1): 17-26

10. Lara MA, Acevedo M, Berenzon S. La depresión femenina vista desde la subjetividad de las mujeres. Cad. Saúde Pública, Río de Janeiro 2004; 20 (3): 818-828.

11. López PA, Lege L. Diferencias entre hombres y mujeres en relación al reconocimiento en sí mismos de sintomatología depresiva. Revista Electrónica de Psicología Iztacala 2009; 12 (1): 32-46.

12. Belló M, Puentes RE, Medina MME; Lozano R. Prevalencia y diagnóstico de depresión en población adulta en México. Salud Pública de México 2005; 47 (1): 4-11.

13. Gómez RC; Bohorquez A, Pinto MD, Gil LJ, Rondón SM, Díaz GN Prevalencia de depresión y factores asociados con ella en la población colombiana. Rev Panam Salud Pública/Pan Am J Public Health 2004; 16 (6): 378-386.

14. Cortés NR. Depresión en estudiantes de enfermería. Avances en psiquiatría biológica 2005; 6: 126-141.

15. Arias GCE. Evaluación de los síntomas depresivos según el inventario de depresión de Beck en los estudiantes universitarios de la Facultad de Farmacia y Bioanálisis. Revista de la Facultad de Farmacia 2004; 46 (2): $16-22$.

16. Moreno CA, Medina M, Icaza ME. Tabaquismo y depresión. Salud Mental 2008; 31: 409-415.

17. Londoño N, Marín C, Juárez F, Palacio J y cols. Factores de riesgo psicosociales y ambientales asociados a trastornos mentales. Suma Psicológica 2010; 17 (1): 59-68.

18. Castillo MRM, Arankowsky SG. Violencia intrafamiliar como factor de riesgo para trastorno depresivo mayor en mujeres: Estudio de casos y controles. Revista Biomédica 2008; 19: 128-136. 